\title{
ESCALA DE INDICADORES POTENCIALES DE LA REPRESENTACIÓN ORGANIZACIONAL: EIPRO
}

\author{
Jesús María de Miguel Calvo ${ }^{1}$
}

El informe de investigación presenta el proceso de construcción de un instrumento de medida para el estudio de la organización como realidad socialmente construida, concretamente las fases para identificar, aislar y discriminar los indicadores del fenómeno que hemos denominado representación organizacional. La delimitación de los indicadores potenciales se ha realizado utilizando técnicas cualitativas (análisis documental, diagrama de afinidad, acuerdo interjueces conceptual).

Los indicadores potenciales pertenecen a cualquiera de las siete dimensiones siguientes: contexto, estructura, procesos organizacionales, clima, cultura, satisfacción, eficacia. El aislamiento y discriminación de los indicadores seleccionados entre los potenciales se ha realizado utilizando técnicas cuantitativas estadísticas (análisis descriptivos, escalamiento multidimensional, análisis factorial e Índices de fiabilidad). Se han seleccionado 49 indicadores que constituyen, a nivel de investigación, las variables independientes utilizadas para el estudio de la organización como construcción social. Su inminente despliegue en elementos (ítemes) medibles (mediante técnicas escalares) constituyen la base informativa del Cuestionario de Representación Organizacional CREOC.

PALABRAS CLAVE: Representación organizacional, construcción social.

The investigation report presents/displays the process of construction of an instrument of measurement for the study of the organization like reality socially constructed, concretely the phases to identify, to isolate and to discriminate the indicators of the phenomenon that we have denominated organizational representation. The boundary of the potential indicators has been made using technical qualitative (documentary analysis, diagram of affinity, agreement in terj udges conceptual-). The potential indicators belong to anyone of the seven following dimensions: organizational context, structure, processes, climate, culture, satisfaction, effectiveness. The isolation and discrimination of the indicators selected between the potentials have been made using technical quantitative statistics (descriptive analyses, multidimensional scaling, factorial analysis and reliability indices). 49 indicators have been selected that constitute, to investigation level, the used independent variables for the study of the organization like social construction. Their imminent unfolding in elements (ítems) measurable (by means of techniques you will climb) constitutes the informative base of the Questionnaire of Representation Organizational CREO@).

KEY WORDS: Organizational representation, social construction

${ }^{1}$ Doctor en Psicología Social. Profesor asociado de la Universidad Autónoma de Madrid en el Centro Universitario de Salud Pública. Dirección profesional: CI General Oraá, 39. 28006 Madrid. España. 


\section{INTRODUCCIÓN}

El artículo trata sobre el problema que suscita el conocimiento socialmente compartido en el seno de las organizaciones sociales y formales. Concretamente al conocimiento acuñado bajo el neologismo Representación Organizacional y definido como: «un sistema de cogniciones y significados elaborados y compartidos por los sujetos de una organización, que funciona como sistema de apropiación, construcción y dominación de la misma. Este sistema se genera en los procesos de interacción y está determinado por la posición que ocupan los sujetos en la organización» (De Miguel, 1999 y De Miguel y Cordero, 2002). Los postulados teóricos del constructo se nutren, fundamentalmente, de las tesis de Berger y Luckman (1968) sobre la construcción social de la realidad y las ideas sobre la representación social de Moscovici (1969) y se desarrollan en De Miguel (1999). Se postula que el fenómeno de la Representación Organizacional tiene poder para describir, explicar y (en cuanto es normativo) predecir el comportamiento de las personas en la organización. Es así ya que mediante este fenómeno las personas interpretan, construyen y organizan modelos de referencia para comprender y valorar la organización y, actuar correctamente cuando se encuentran bajo su influencia. El interés de la investigación se centra en como las personas crean y recrean el conocimiento de la organización como realidad socialmente construida. Se insiste en la importancia de la interacción como proceso fundamental tanto para acceder como para generar conocimiento. Se atiende, especialmente, a esas interacciones impuestas formalmente por la posición que ocupa la persona en función de su rol organizacional y, en definitiva facilitan y/o restringen el acceso al conocimiento.

El objetivo de investigación presentado en las páginas que siguen consiste en identificar y aislar los indicadores susceptibles de formar parte de la representación organizacional $y$, supone una fase inicial de investigación de mayor envergadura.

Tabla 1. Distribución y descripción de los sujetos.

\begin{tabular}{|l|c|c|}
\hline \multicolumn{1}{|c|}{ GRUPOS } & N & $\mathbf{\%}$ \\
\hline $\begin{array}{l}\text { GRUPO 1. Directivos y mandos: el criterio utilizado fue el de personas que ocuparan } \\
\text { puestos de responsabilidad directiva y que no estuvieran regidos por el convenio } \\
\text { laboral. Al seleccionar este colectivo se pretende tener en cuenta la visión de la } \\
\text { empresa u organización. El directivo o mando fuera de convenio, que pacta su relación } \\
\text { contractual con la organización, es el garante de que se cumplan los objetivos de la } \\
\text { organización y asume la labor de transmisión de los procesos instituyentes }\end{array}$ & 20 & 33 \\
\hline $\begin{array}{l}\text { GRUPO 2. Académicos: sujetos que trabajan en el ámbito teórico o de investigación } \\
\text { en el ámbito académico: universidades, escuelas de negocios, etcétera. }\end{array}$ & 20 & 33 \\
\hline $\begin{array}{l}\text { GRUPO 3. Consultores: sujetos que trabajan realizando tareas de asesoramiento y/o } \\
\text { consultoría como profesionales externos a la organización. }\end{array}$ & 20 & 33 \\
\hline $\begin{array}{l}\text { GRUPO 4. Técnicos: sujetos que trabajan en Direcciones o Departamentos de } \\
\text { Organización y Recursos Humanos, en Direcciones de Personal o cualesquiera otras } \\
\text { que desarrollen funciones «tecnoestructurales» relacionadas con las funciones ya } \\
\text { indicadas. }\end{array}$ & 20 & 33 \\
\hline $\begin{array}{l}\text { GRUPO 5. Representantes de los trabajadores: han participado miembros de los } \\
\text { sindicatos UGT, CCOO y CS1-CSIF y miembros independientes de comités de } \\
\text { empresa. Alguno de los participantes de UGT y CCOO fueron personas que, a juicio } \\
\text { de sus centrales sindicales respectivas, eran expertas en materias de organización y de } \\
\text { recursos humanos. }\end{array}$ & 20 & 33 \\
\hline
\end{tabular}




\section{MÉTODO}

\section{Sujetos}

165 personas participaron en la identificación de los elementos o indicadores de la representación organizacional y actuaron como jueces. Fueron seleccionadas por ser expertos en «Organización y Recursos Humanos» y como tal, se les suponen las competencias necesarias para realizar la función de juez en esta tarea. Los 165 jueces $^{2}$ se distribuían homogéneamente en cinco grupos como se describe en la tabla 1.

Instrumento: ESCALA DE INDICADORES POTENCIALES DE LA REPRESENTACIÓN SOCIAL (EIPRO). El instrumento de recogida de información (EIPRO) es una escala de aplicación autoadministrada que incluye 79 elementos (tabla 2): Para construir esta escala se preseleccionaron elementos de aspectos que resultan especialmente relevantes para los sujetos de la organización. Estos aspectos tienen que ver con: 1.- las dimensiones que configuran y determinan la realidad organizacional: contexto, estructura y procesos. 2.-las dimensiones que evidencian la experiencia psicológica o psicosocial de la realidad organizacional: clima y cultura. 3.- las dimensiones que muestran los resultados de la organización: satisfacción y eficacia. Se identificaron, pues, siete dimensiones y se detallaron sus variables constituyentes a partir de fuentes bibliográficas y documentales de autores prestigiados en cada materia3; generando un inventario de más de 300 indicadores potenciales de la representación organizacional. Estas unidades de análisis se agruparon mediante un diagrama de afinidad. Para realizar el diagrama se diseñó un sistema de fichas. Cada una de ellas contenía el término conceptual de la unidad de análisis y su descripción. Las fichas se fueron ordenando en una gran pantalla de visualización, agrupándolas en función de su coherencia conceptual. Tres jueces expertos realizaron y revisaron las agrupaciones hasta llegar al acuerdo. Las agrupaciones formadas atendieron al significado y sentido de las definiciones conceptuales y no a su denominación terminológica. Se pretendía homogeneizar los significados y reducir la dispersión de los significantes.

2 Los sujetos que participaron en la fase experimental pertenecían a alguna de las organizaciones o instituciones presentadas a continuación. No están representadas todas las organizaciones ya que cierto número de expertos prefirieron mantener su organización en el anonimato y se respeta su opción: AEA; Alcatel Standard Eléctrica; Alta Gestión S.A.;Andersen Consulting; ATT; Ayuntamiento de Tres Cantos; Berde Consultores; Caja de Madrid; Centro Universitario de Investigación y Formación Empresarial-CUIFE(UAM); Centro Universitario de Salud Pública -CUSP- (UAM); CEPSA; Confederación de sindicatos independientes y sindical de funcionarios -CSI-CSIF-; Consultores de organización -CONORG-; Comisiones Obreras -CCOO-; Coopers \& Librand; Corporación Ibérica de Nutrición; DElRIS, S.A.; Desarrollo Organizacional (0.0.); Empresa Nacional del Uranio, S.A: -ENUSA-; Escuela de organización Industrial-EOI; Facultad de Ciencias Económicas y Empresariales (UCM); Facultad de Psicología (UAM); FENOSA; Formación y Consultoría S.A. -FYCSA-; Gestevisión Telecinco S.A.; Granada Computer Services; Grupo lnmark; Hewlett Packard Española, S.A.; IBM; IDEA (Instituto de estudios Aplicados); INNOVA (Gestión de la Innovación); Instituto de Administración de Empresas -IADE(UAM); Instituto de Estudios Aplicados IDEA-; INTERSOC; Programa de Cooperación Educativa en O+RH (UAM); Renault España Comercial; SEUR (Servicio de transporte urgente); Tabacalera; TEA Cegos; Tecknowledge; Telefónica; TNT Express Worldwide; Unangel Consultores; Unión General de Trabajadores- UGT-; Universidad de Salamanca; $\begin{array}{llll}\text { Universidad San } & \text { Pablo } & \text { CEU; } & \text { Valeo. }\end{array}$ 
Tabla 2. Inventario de indicadores potenciales de la representación organizacional.

\begin{tabular}{|c|c|c|c|}
\hline 1. Absentismo & 21. Confort & 41. Flexibilidad/ Innovación & 61. Presión \\
\hline 2. Accidentabilidad & 22. Consenso en los objetivos & 42. Flexibilidad/ Tradicionalismo & $\begin{array}{l}\text { 62. Presunciones Básicas } \\
\text { Subyacentes }\end{array}$ \\
\hline $\begin{array}{l}\text { 3. Administración de } \\
\text { Políticas }\end{array}$ & 23. Control & $\begin{array}{l}\text { 43. Grado de Estructura Impuesta } \\
\text { sobre la Posición Ocupada }\end{array}$ & 63. Productividad \\
\hline 4. Agrupación de Unidades & 24. Cooperación & $\begin{array}{l}\text { 44. Habilidades de Trabajo de la } \\
\text { Dirección }\end{array}$ & 64. Profesionalización \\
\hline 5. Amplitud de Control & 25. Coordinación & $\begin{array}{l}\text { 45. Habilidades Interpersonales } \\
\text { de la Dirección }\end{array}$ & 65. Propiedad y Control \\
\hline 6. Amplitud Vertical & 26. Crecimiento & 46. Identidad & 66. Relaciones sociales \\
\hline 7. Aроуо & 27. Delegación & 47. Implicación & 67. Retribución \\
\hline 8. Autonomía & $\begin{array}{l}\text { 28. Desarrollar Personas y } \\
\text { Equipos }\end{array}$ & 48. Iniciativa & 68. Rotación \\
\hline 9. Beneficio & 29. Dirección & 49. Interacción e Influencia & 69. Satisfacción en el Trabajo \\
\hline 10.Calidad & 30. Eficiencia & $\begin{array}{l}\text { 50. Interdependencia con otras } \\
\text { Organizaciones }\end{array}$ & 70. Sistema de Recompensa \\
\hline 11. Capacitación & 31. Énfasis en el Logro & $\begin{array}{l}\text { 51. Internalización de objetivos } \\
\text { de la Organización }\end{array}$ & 71. Tamaño \\
\hline 12. Cana de la Compañía & 32. Equidad & 52. Localización & 72. Tamaño de la Unidad \\
\hline 13. Centralización & $\begin{array}{l}\text { 33. Especialización del } \\
\text { Trabajo }\end{array}$ & $\begin{array}{l}\text { 53. Metas de Desempeño y } \\
\text { Entrenamiento }\end{array}$ & 73. Tecnología \\
\hline 14. Claridad & 34. Estabilidad & 54. Moral & 74. Tolerancia al Conflicto \\
\hline 15.Cohesión & 35. Establecimiento de Metas & 55. Motivación & 75. Tolerancia del Riesgo \\
\hline 16. Complejidad & 36. Estandarización & 56. Organizar & 76. Toma de Decisiones \\
\hline $\begin{array}{l}\text { 17.Componente } \\
\text { Administrativo }\end{array}$ & 37. Estatus & $\begin{array}{l}\text { 57. Orientación hacia la } \\
\text { Recompensa }\end{array}$ & 77. Utilización del entorno \\
\hline 18. Comunicación & 38. Estructura & 58. Origen e historia & $\begin{array}{l}\text { 78. Valor de los Recursos } \\
\text { Humanos }\end{array}$ \\
\hline 19. Configuración & $\begin{array}{l}\text { 39. Evaluación del } \\
\text { Rendimiento }\end{array}$ & $\begin{array}{l}\text { 59. Participación e Influencia } \\
\text { Compartida }\end{array}$ & $\begin{array}{l}\text { 79. Valoración por Entidades } \\
\text { Externas }\end{array}$ \\
\hline 20. Conflicto & 40. Flexibilidad/ Adaptación & 60. Planificar & \\
\hline
\end{tabular}


El instrumento, denominado «Escala de indicadores potenciales de la representación organizacional» incluye los 79 indicadores preseleccionados e inventariados (tabla 2) y se estructura en tres partes: portada, instrucciones y escala. La escala se diseñó con un formato a tres columnas. La primera columna correspondía al concepto analizado, la segunda a la descripción del concepto (con ello se reduce la ambigüedad característica de muchos de los conceptos) y, la tercera a la escala numérica, de 1 a 10, donde se debería indicar la puntuación atribuida a cada concepto. Una última página incluida dos preguntas abiertas (sobre elementos que, pudiendo ser importantes, no se habían considerado y sobre observaciones que a juicio del experto se pudieran hacer sobre el estudio) y los datos del experto. Todas las páginas están encabezadas con la instrucción: «Cree usted que el concepto ( ... ) es importante en la imagen que tienen de una empresa sus empleados».

\section{PROCEDIMIENTO DE RECOGIDA DE DATOS}

Se solicitó a los sujetos participantes su opinión de experto sobre el grado en que tales conceptos forman parte de la imagen que tienen los trabajadores de su organización. Tal opinión debían expresarla en función del «grado de importancia» en una escala de 1 a 10. La estrategia para recoger los protocolos se diversificó en las siguientes fórmulas de actuación: 1.- Reunión con los directivos de la empresa donde se desarrolló la primera fase de la investigación. Dichos profesionales fueron seleccionados y convocados por la Dirección de Recursos Humanos de la empresa. 2.-Solicitud de entrevistas con diferentes expertos a través de contacto personal o a través de terceras personas que sirvieron de intermediario. 3.- Expertos captados en diversas acciones formativas desarrolladas por el autor del presente trabajo. 4.-Reuniones con personas de los sindicatos mayoritarios en el territorio español, quienes seleccionaron y remitieron los protocolos a representantes de los trabajadores que cumplieran las características de «experto» anteriormente indicadas. 5.- Envío postal de protocolos utilizando las bases de datos de organizaciones que vinculan el mundo académico con el empresarial4 - A las personas que contestaron los protocolos siguiendo las formulas 1, 2 y 3 se les explicó personalmente el objetivo, características y alcance de la investigación y, en particular, del instrumento. Aquellos que contestaron bajo las fórmulas 4 y 5 dispusieron de una página explicativa de estos aspectos añadida al protocolo. La participación de los expertos, exceptuando aquellos incluidos en el punto uno, fue completamente voluntaria. En cuanto a los expertos del grupo uno, si bien su participación no fue obligatoria, tampoco puede afirmarse que fuera estrictamente voluntaria.

\section{TRATAMIENTO DE DATOS}

La discriminación de los indicadores relevantes para el estudio de la representación organizacional se ha llevado a cabo mediante el análisis de la dimensión «importancia», dimensión cuestionada en la Escala. Este análisis se completa con la validación empírica de las siete dimensiones teóricas que sirvieron como fuente para seleccionar las variables de la escala. El análisis se ha realizado en función de cuatro criterios: Atribución: puntuación media asignada a las variables por los expertos. Pertenencia: el indicador se incluye estadísticamente en la dimensión importancia - «Es importante en la imagen de la empresa»-. Explicación: porcentaje de varianza explicada por la variable en la dimensión teórica. Fiabilidad: incidencia de la variable en el Índice de fiabilidad de la dimensión teórica.

Con intención de recabar datos relativos a estos criterios se ha procedido a realizar una serie de análisis estadísticos. Los análisis estadísticos se realizaron utilizando el paquete estadístico SPSS para Windows: El análisis descriptivo se solicitaron los siguientes estadísticos: tablas de frecuencias, rango, valores mínjmo y máximo, media, desviación típica, percentil 25 y número de sujetos que contestaron a cada una de las preguntas. Se procedió a realizar un escalamiento multidimensional con la intención de separar los indicadores que pertenecen a la dimensión «importancia» de los que no han sido así considerados por los expertos. Análisis factoriales. Previamente al análisis de las dimensiones se contrastó la adecuación del análisis mediante la prueba de adecuación muestral (Kaiser-Meyer-Olkin) y la prueba de esfericidad de Bartlett. 
Contrastada la adecuación del análisis se procedió a su ejecución. Para la extracción de factores se seleccionó el método de ejes principales, que utiliza la proporción de varianza explicada reproducida por los factores comunes. Se optó por la extracción de un único factor ya que nuestro objetivo era conocer qué proporción de varianza explicada correspondía a cada una de las variables que componían una dimensión. Al tratarse de un único factor no se solicitó ningún método de rotación. En el análisis de la fiabilidad se utilizó el coeficiente alfa de Cronbach, solicitando los estadísticos de la dimensión en su conjunto y los coeficientes alfa de la dimensión prescindiendo de cada una las variables (de esta forma se comprueba si el índice de fiabilidad aumenta o se reduce suprimiendo cada una de las variables en el conjunto de la escala). 
Tabla 3. Criterios de decisisón para la selección de los indicadores y criterios de valoración empírica de las dimensiones teóricas.

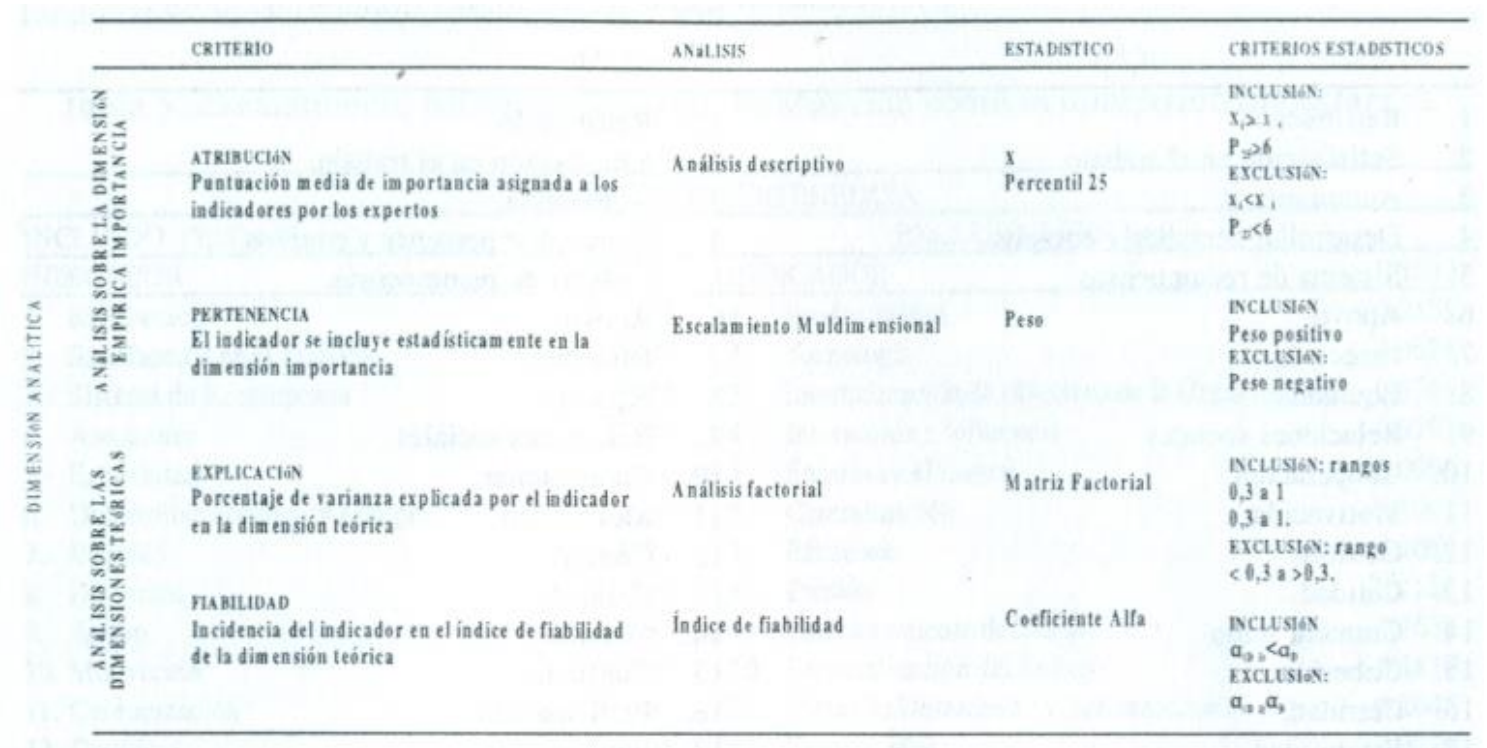

\section{RESULTADOS}

El análisis estadístico de los criterios utilizados para seleccionar los indicadores de la representación organizacional aportan los siguientes resultados:

Criterio 1. Atribución: la discriminación de los indicadores relevantes atendiendo a la importancia atribuida por los expertos a los indicadores potenciales se ha realizado considerando la media de las puntuaciones y el percentil 25 (la puntuación en el percentil 25 indica que el $75 \%$ de los expertos ha valorado al indicador de que se trate igualo por encima de tal puntuación). Los criterios de decisión para evaluar la inclusión/exclusión de los respectivos indicadores han sido los siguientes:

Criterios de inclusión:

1. $\mathrm{x}_{\mathrm{i}}>\mathrm{x}_{\mathrm{j}}$ (siendo $\mathrm{X}_{\mathrm{i}}$, la puntuación media del indicador $\mathrm{y} \mathrm{x}$, la media de los 79 indicadores)

2. $\mathrm{P}_{25}>6$ (siendo $\mathrm{P}_{25}$,el percentil 25)

Criterios de exclusión:

$\mathrm{x}_{\mathrm{i}}<\mathrm{x}_{\mathrm{j}}$

$\mathrm{P}_{25}<6$

El listado de indicadores que cumplen con alguno de los dos criterios de inclusión se presenta en la tabla 4. Como se puede apreciar existe una gran coincidencia entre los elementos seleccionados bajo ambos criterios. Únicamente cuatro de esos elementos no han sido seleccionados por ambos 
Tabla 4. Listado de indicadores seleccionados en función del criterio «Atribución».

\begin{tabular}{|c|c|}
\hline \multicolumn{2}{|c|}{ Criterio de atribución } \\
\hline$\left({ }_{i} 3_{t}\right)$ & $\left(\mathrm{P}_{25}^{3} 6\right)$ \\
\hline $\begin{array}{l}\text { 1. Retribución. } \\
\text { 2. Satisfacción en el trabajo. } \\
\text { 3. Autonomía. } \\
\text { 4. Desarrollar personas y equipos. } \\
\text { 5. Sistema de recompensas. } \\
\text { 6. Apoyo. } \\
\text { 7. Dirección. } \\
\text { 8. Equidad. } \\
\text { 9. Relaciones sociales. } \\
\text { 10. Cooperación. } \\
\text { 11. Motivación. } \\
\text { 12. Confort } \\
\text { 13. Calidad. } \\
\text { 14. Comunicación. } \\
\text { 15. Cohesión. } \\
\text { 16. Claridad. } \\
\text { 17. Estabilidad. } \\
\text { 18. Conflicto. } \\
\text { 19. Participación. } \\
\text { 20. Implicación. } \\
\text { 21. Organizar. } \\
\text { 22. Iniciativa } \\
\text { 23. Toma de decisiones. } \\
\text { 24. Capacitación. } \\
\text { 25. Orientación hacia la recompensa. } \\
\text { 26. Identidad. } \\
\text { 27. Valor de los recursos humanos. } \\
\text { 28. Beneficio. } \\
\text { 29. Evaluación del rendimiento. } \\
\text { 30. Coordinación. } \\
\text { 31. Habilidades Interpersonales de la dirección. } \\
\text { 32. Habilidades de Trabajo de la dirección } \\
\text { 33. Planificar. } \\
\text { 34. Accidentabilidad. } \\
\text { 35. Administración de políticas } \\
\text { 36. Crecimiento. } \\
\text { 37. Tecnología. } \\
\text { 38. Delegación. } \\
\text { 39. Profesionalización. } \\
\text { 40. Flexibilidad/innovación. } \\
\text { 41. Flexibilidad/adaptación. } \\
\text { 42. Productividad. } \\
\text { 43. Valoración por entidades externas. } \\
\text { Moral. }\end{array}$ & $\begin{array}{l}\text { 1. Retribución. } \\
\text { 2. Satisfacción en el trabajo. } \\
\text { 3. Autonomía. } \\
\text { 4. Desarrollar personas y equipos. } \\
\text { 5. Sistema de recompensas. } \\
\text { 6. Apoyo. } \\
\text { 7. Dirección. } \\
\text { 8. Equidad. } \\
\text { 9. Relaciones sociales. } \\
\text { 10. Cooperación. } \\
\text { 11. Motivación. } \\
\text { 12. Confort } \\
\text { 13. Calidad. } \\
\text { 14. Comunicación. } \\
\text { 15. Cohesión. } \\
\text { 16. Claridad. } \\
\text { 17. Estabilidad. } \\
\text { 18. Conflicto. } \\
\text { 19. Participación. } \\
\text { 20. Implicación. } \\
\text { 21. Organizar. } \\
\text { 22. Iniciativa } \\
\text { 23. Toma de decisiones. } \\
\text { 24. Capacitación. } \\
\text { 25. Orientación hacia la recompensa. } \\
\text { 26. Identidad. } \\
\text { 27. Valor de los recursos humanos. } \\
\text { 28. Beneficio. } \\
\text { 29. Evaluación del rendimiento. } \\
\text { 30. Coordinación. } \\
\text { 31. Habilidades Interpersonales de la dirección. } \\
\text { 32. Habilidades de Trabajo de la dirección } \\
\text { 33. Planificar. } \\
\text { 34. Accidentabilidad. } \\
\text { 35. Administración de políticas } \\
\text { 36. Crecimiento. } \\
\text { 37. Tecnología. } \\
\text { 38. Delegación. } \\
\text { 39. Profesionalización. } \\
\text { 40. Flexibilidad/innovación. } \\
\text { 41. Flexibilidad/adaptación. } \\
\text { 42. Productividad. } \\
\text { 43. Valoración por entidades externas. Moral. } \\
\text { 44. Absentismo } \\
\text { 45. Tolerancia al conflicto } \\
\text { 46. Grado de estructura impuesta sobre lasión ocupada } \\
\text { posición }\end{array}$ \\
\hline
\end{tabular}


Criterio 2. Pertenencia: Se ha utilizado el escalamiento multidimensional como instrumento para evaluar el grado de ajuste al criterio «pertenencia». El criterio de inclusión establecido para determinar la selección de indicadores ha sido: Peso $>0$.

El criterio de exclusión ha sido, por tanto:

Peso; < 0 . Según se aprecia en la tabla 5, hay 46 indicadores que presentan un peso positivo en la dimensión importancia y por ello han sido incluidos en la lista de elementos seleccionados por el criterio pertenencia.

Tabla 5. Escalamiento Multidimensional. Puntuación sobre la dimensión importancia.

\begin{tabular}{|c|c|c|c|}
\hline \multicolumn{4}{|c|}{ CRITERIO PERTENENCIA } \\
\hline Incluido: peso $>0$ & & Excluido: peso $<0$ & \\
\hline Indicador & peso & Indicador & peso \\
\hline Retribución & 1,5513 & Productividad & $-0,0135$ \\
\hline Satisfacción en el Trabajo & 1,5487 & Tecnología & $-0,0624$ \\
\hline Sistema de Recompensa & 1,4984 & Internalización de objetivos de la Organización & $-0,0745$ \\
\hline Autonomía & 1,3614 & Interacción e Influencia & $-0,0793$ \\
\hline Estabilidad & 1,3511 & Énfasis en el Logro & $-0,0999$ \\
\hline Desarrollar Personas y Equipos & 1,3220 & Centralización & $-0,1001$ \\
\hline Equidad & 1,3171 & Eficiencia & $-0,1085$ \\
\hline Dirección & 1,1895 & Presión & $-0,1124$ \\
\hline Apoyo & 1,1635 & Establecimiento de Metas & $-0,1638$ \\
\hline Motivación & 1,0923 & Especialización del Trabajo & $-0,1819$ \\
\hline Comunicación & 1,0437 & Metas de Desempeño y Entrenamiento & $-0,2045$ \\
\hline Conflicto & 1,0185 & Complejidad & $-0,3223$ \\
\hline Cooperación & 0,9428 & Estructura & $-0,4883$ \\
\hline AccidentabiJidad & 0,9136 & Tolerancia del Riesgo & $-0,5110$ \\
\hline Relaciones sociales & 0,8752 & Rotación & $-0,5502$ \\
\hline Calidad & 0,8677 & Control & -06526 \\
\hline Participación e Influencia Compartida & 0,8500 & Utilización del entorno & $-0,6930$ \\
\hline Confort & 0,8341 & Flexibilidadl Tradicionalismo & $-0,7611$ \\
\hline Implicación & 0,8132 & Estatus & $-0,8138$ \\
\hline Claridad & 0,8067 & Interdependencia con otras Organizaciones & $-0,8266$ \\
\hline Cohesión & 0,7586 & Estandarización & $-0,8723$ \\
\hline Iniciativa & 0,7404 & Configuración & $-1,0537$ \\
\hline Identidad & 0,7263 & Amplitud Vertical & $-1,3526$ \\
\hline Beneficio & 0,6880 & Carta de la Compañía & $-1,5443$ \\
\hline Organizar & 0,6669 & Propiedad y Control & $-1,5553$ \\
\hline Valor de los Recursos Humanos & 0,6634 & Amplitud de Control & $-1,5682$ \\
\hline Toma de Decisiones & 0,6207 & Agrupación de Unidades & $-1,5824$ \\
\hline Habilidades de Trabajo de la Dirección & 0,5482 & Componente Administrativo & $-1,7591$ \\
\hline Orientación hacia la Recompensa & 0,5258 & Presunciones Básicas Subyacentes & $-1,9420$ \\
\hline Evaluación del Rendimiento & 0,4754 & Tamaño & $-2,1829$ \\
\hline Capacitación & 0,4232 & Localización & $-2,2958$ \\
\hline Flexibilidadl Adaptación & 0,3755 & Tamaño de la Unidad & $-2,4956$ \\
\hline Consenso en los objetivos & 0,3552 & Origen e historia & $-2,7434$ \\
\hline Flexibilidadl Innovación & 0,2871 & & \\
\hline Habilidades Interpersonales de la Dirección & 0,2790 & & \\
\hline Administración de Políticas & 0,2679 & & \\
\hline Crecimiento & 0,2666 & & \\
\hline Coordinación & 0,2577 & & \\
\hline Moral & 0,1716 & & \\
\hline Planificar & 0,1397 & & \\
\hline Tolerancia al Conflicto & 0,1276 & & \\
\hline Delegación & 0,0788 & & \\
\hline Absentismo & 0,0295 & & \\
\hline Valoración por Entidades Externas . & 0,0269 & & \\
\hline Profesionalización & 0,0202 & & \\
\hline Grado de Estructura Impuesta sobre la Posición & 0,0083 & & \\
\hline Ocupada & & & \\
\hline
\end{tabular}


Criterios 3 y 4: Explicación y fiabilidad. Los análisis realizados en este punto se realizan sobre las siete dimensiones teóricas de la representación organizacional y sirven para contrastar si estas siete dimensiones están construidas con variables coherentes. La coherencia viene determinada, por un lado, por su propia pertinencia teórica (validez de constructo) y, por otro, por su adecuación a la percepción empírica de importancia de los sujetos expertos evaluadores (validez empírica).

Para evaluar el criterio «explicación» se consideró pertinente realizar un análisis factorial con la premisa inicial de que todos los indicadores pertenecían a una dimensión. Por ello, se forzó en el análisis la extracción de un único factor, de esta manera se comprobaría en la matriz factorial el peso explicativo del indicador en el porcentaje total de varianza explicada en la dimensión. El criterio de exclusión cuantificado rechazaría dentro de la dimensión aquellos indicadores cuyo peso estuviera en el rango de $<0,3$ a $>-0,3$. El criterio de inclusión afectará, por tanto, a los rangos 0,3 a 1 y de $-0,3$ a -1 .

El criterio «fiabilidad» persigue determinar en qué medida se relacionan los elementos de la escala de indicadores potenciales de la representación organizacional. De tal forma que se puedan excluir de la dimensión aquellos elementos que manifiesten poca relación con el resto de los indicadores de la escala. El estadístico que se utilizó fue el coeficiente "Alfa de Cronbach» (es un índice de consistencia interna basado en el promedio de correlación entre-elementos). Se ha obtenido tanto el índice de fiabilidad global para el conjunto de indicadores de cada dimensión, como los coeficientes alfa que podrían alcanzarse si se optara por eliminar cada uno de los indicadores específicos que componen la dimensión teórica. De esta forma, es factible comparar el índice de fiabilidad global de la dimensión con las variaciones experimentadas en el mismo, al ir eliminando indicadores. El criterio matemático utilizado para rechazar los elementos poco relacionados consistió en excluir aquellos que, en el caso de eliminarse de la escala, no hicieran variar el índice de fiabilidad o determinasen el aumento del mismo.

Criterio de exclusión $=\mathfrak{a}_{(\mathrm{D}-\mathrm{i})}{ }^{3} \mathrm{a}_{\mathrm{D}}$

Criterio de inclusión $=a_{(D-i)}<a_{D}$

\section{Dimensión 1. Contexto}

Antes de exponer los resultados alcanzados en dicha dimensión con relación a los criterios de explicación y de fiabilidad, conviene mencionar los valores descriptivos logrados por el conjunto de indicadores que la integran. Frente a una puntuación media del conjunto de variables ()$=6,86$ y una puntuación media de las medias de las dimensiones $(\mathrm{d})=6,90$, la media de los siete indicadores de la dimensión contexto se sitúa en 5,68. Cinco de los siete indicadores que pertenecen a esta dimensión se incluyen entre los diez indicadores con puntuaciones más bajas en el grado de importancia atribuido por los expertos. Sólo uno de estos siete indicadores, «tecnología» $(=7,01)$ se considera importante según los criterios de atribución y pertenencia; Los análisis factoriales y de fiabilidad indican que la dimensión teórica posee una estructura fuerte. Ningún elemento queda excluido. Véanse tablas 6 y 7. 
Tabla 6. Contexto. Matriz Factorial.

\begin{tabular}{|l|l|l|}
\hline & MATRIZ FACTORIAL & COMUNALIDADES \\
\hline Interdependencia con otras Organizaciones & 0,74317 & 0.48445 \\
Origen e historia & 0,68022 & 0,36600 \\
Localización & 0,64151 & 0,30448 \\
Tamaño & 0,63156 & 0,28517 \\
Propiedad y Control & 0,59057 & 0,24392 \\
Carta de la Compañía & 0,52541 & 0,18191 \\
Tecnología & 0,51922 & 0,17466 \\
\hline Porcentaje de Varianza explicada & 38,9 & 29,2 \\
\hline
\end{tabular}

Tabla 7.- Contexto. Índice de Fiabilidad.

\begin{tabular}{|l|l|l|}
\hline \multicolumn{1}{|c|}{ INDICADOR } & \multicolumn{1}{c|}{$\begin{array}{c}\text { CORRELACIÓN } \\
\text { INDICADOR TOTAL }\end{array}$} & $\begin{array}{c}\text { ALFA } \\
\text { PRESCINDIENDO } \\
\text { DEL INDICADOR }\end{array}$ \\
\hline Interdependencia con otras Organizaciones & 0,5723 & 0,6717 \\
Origen e historia & 0,4990 & 0,6889 \\
Localización & 0,4683 & 0,6968 \\
Tamaño & 0,4600 & 0,6987 \\
Propiedad y Control & 0,4169 & 0,7087 \\
Carta de la Compañía & 0,3570 & 0,7210 \\
Tecnología & 0,3546 & 0,7245 \\
\hline N de casos válidos: 162 & \\
N de indicadores: $\quad 7$ & \\
Alfa $\quad 0,7334$ & \\
\hline
\end{tabular}

En cuanto al criterio de «explicación», todos los indicadores tienen peso superior a 0,5 , suficiente para considerar adecuada su inclusión en dicha dimensión. En cuanto al criterio «fiabilidad», suprimiendo cualquiera de los indicadores, el coeficiente alfa perdería valor. De ahí que todos los indicadores resulten pertinentes en la dimensión.

Dimensión 2. Estructura.

De forma general se puede concluir que los expertos que contestaron ara escala, atribuyen a la dimensión estructura menor importancia que al resto de las dimensiones. $(=6.23)$, sí se exceptúa la dimensión contexto. De los catorce indicadores que constituyen esta dimensión, sólo tres superan los criterios previos de atribución y de pertenencia: «autonomía», «capacitación» y «profesionalización».

Los resultados obtenidos con relación al criterio de explicación, ponen en evidencia que de los catorce indicadores dos de ellos quedan excluidos. Dichos indicadores son: «autonomía» y «capacitación». La matriz factorial nos informa de que no llegan a explicar más del $30 \%$ de la varianza del factor (tabla 8). El índice de fiabilidad más elevado' es el que integra los catorce indicadores. No obstante, si se eliminaran estos indicadores, el Índice de fiabilidad se vería escasamente afectado (tabla 9). 
Tabla 8. Estructura. Matriz Factorial.

\begin{tabular}{|l|l|l|}
\hline & MATRIZ & COMUNALIDADES \\
& FACTORIAL & \\
\hline Agrupación de Unidades & 0,57 & 0,32 \\
Estandarización & 0,56 & 0,31 \\
Especialización del Trabajo & 0,55 & 0,30 \\
Componente Administrativo & 0,55 & 0,30 \\
Complejidad & 0,53 & 0,28 \\
Centralización & 0,52 & 0,28 \\
Configuración & 0,48 & 0,23 \\
Amplitud de Control & 0,45 & 0,21 \\
Flexibilidad/Tradicionalismo & 0,45 & 0,20 \\
Tamaño de la Unidad & 0,41 & 0,16 \\
Amplitud Vertical & 0,40 & 0,16 \\
Profesionalización & 0,31 & 0,10 \\
Autonomía & $<0,3$ & 0,09 \\
Capacitación & $<0,3$ & 0,07 \\
\hline Porcentaje de Varianza explicada & 26,9 & 21,6 \\
\hline
\end{tabular}

Tabla 9. Estructura. Índice de Fiabilidad.

\begin{tabular}{|l|l|l|}
\hline \multicolumn{1}{|c|}{ INDICADOR } & $\begin{array}{c}\text { CORRELACIÓN } \\
\text { INDICADOR } \\
\text { TOTAL }\end{array}$ & $\begin{array}{c}\text { ALFA } \\
\text { PRESCINDIENDO } \\
\text { DEL INDICADOR }\end{array}$ \\
\hline Agrupación de Unidades & 0,51 & 0,75 \\
Estandarización & 0.50 & 0,75 \\
Especialización del Trabajo & 0.48 & 0,76 \\
Componente Administrativo & 0.46 & 0,76 \\
Complejidad & 0.44 & 0,76 \\
Centralización & 0.42 & 0,76 \\
Configuración & 0.41 & 0,76 \\
Amplitud de Control & 0.39 & 0,76 \\
Flexibilidad/Tradicionalismo & 0.36 & 0,77 \\
Tamaño de la Unidad & 0.34 & 0,77 \\
Amplitud Vertical & 0.33 & 0.77 \\
Profesionalización & 0.29 & 0,77 \\
Autonomía & 0.28 & 0,77 \\
Capacitación & 0.27 & 0,77 \\
\hline N de casos válidos: $\quad 162$ & \multicolumn{2}{|l}{} \\
N de indicadores: $\quad 7$ & \multicolumn{2}{|c|}{ Alfa }
\end{tabular}

\section{Dimensión 3. Procesos Organizacionales.}

Según los resultados obtenidos en los criterios de «pertenencia» y de «atribución», de los catorce indicadores que componen esta dimensión, diez de ellos satisfacen ambos. Así mismo, la media de las medias de los indicadores de esta dimensión arroja un valor de 7,15. Puntuación que ubica a la dimensión Procesos Organizacionales en una posición intermedia de importancia dentro del conjunto de dimensiones consideradas. Atendiendo al criterio «explicación» se observa que el indicador «Control» presenta una saturación inferior a 0,30 (tabla 10). 
Tabla 10. Procesos Organizacionales. Matriz Factorial.

\begin{tabular}{|l|l|l|}
\hline & $\begin{array}{c}\text { MATRIZ } \\
\text { FACTORIAL }\end{array}$ & COMUNALIDADES \\
\hline Dirección & 0,75 & 0,56 \\
Comunicación & 0,66 & 0,43 \\
Coordinación & 0,66 & 0,43 \\
Delegación & 0,65 & 0,42 \\
Interacción e Influencia Establecimiento de & 0,64 & 0,42 \\
Metas & 0,62 & 0,38 \\
Metas de Desempeño y Entrenamiento & 0,62 & 0,38 \\
Planificar & 0,56 & 0,32 \\
Evaluación del Rendimiento & 0,54 & 0,29 \\
Toma de Decisiones & 0,53 & 0,29 \\
Motivación & 0,51 & 0,26 \\
Organizar & 0,50 & 0,25 \\
Administración de Políticas & 0,49 & 0,24 \\
Control & $<0,3$ & 0,06 \\
\hline Porcentaje de Varianza explicada & 38,2 & 33,8 \\
\hline
\end{tabular}

Los resultados obtenidos con relación al criterio de «fiabilidad», subrayan que el Índice de fiabilidad conjunta de la dimensión se incrementa de 0,8730 a 0,8811 (tabla 11) si se excluyera el indicador «Control». Por lo tanto, «Control» queda excluido de esta dimensión.

Tabla 11. Procesos Organizacionales. Índice de Fiabilidad.

\begin{tabular}{|l|l|l|}
\hline \multicolumn{1}{|c|}{ INDICADOR } & $\begin{array}{c}\text { CORRELACIÓN } \\
\text { INDICADOR } \\
\text { TOTAL }\end{array}$ & $\begin{array}{c}\text { ALFA PRESCINDIENDO } \\
\text { DEL INDICADOR }\end{array}$ \\
\hline Dirección & 0,70 & 0,86 \\
Comunicación & 0,64 & 0,86 \\
Coordinación & 0,62 & 0,86 \\
Delegación & 0,61 & 0,86 \\
Interacción e Influencia Establecimiento de Metas & 0,59 & 0,86 \\
Metas de Desempeño y Entrenamiento & 0,58 & 0,86 \\
Planificar & 0,57 & 0,86 \\
Evaluación del Rendimiento & 0,53 & 0,86 \\
Toma de Decisiones & 0,53 & 0,86 \\
Motivación & 0,50 & 0,87 \\
Organizar & 0,48 & 0,87 \\
Administración de Políticas & 0,47 & 0,87 \\
Control & 0,46 & 0,87 \\
\multicolumn{2}{|c|}{156} & 0,24 \\
\hline$N$ de casos válidos: $\quad, 8730$ & \\
Alfa indicadores: 14 & \\
\hline
\end{tabular}

Dimensión 4. Clima Laboral.

Esta dimensión obtiene un valor de 7,37 en la media de las medias de los indicadores que la integran. De los 19 indicadores de esta dimensión, 14 de ellos cumplen alguno de los dos criterios de «atribución» y de «pertenencia». La utilización del criterio de «explicación» pone en evidencia que los indicadores «Control» $\mathrm{y}$ «Estatus» no cumplen con el criterio de inclusión establecido (tabla 12). Por su parte, el Índice de «fiabilidad» aumenta si se suprimen cualquiera de los indicadores «Control» $\mathrm{y}$ «Estatus» (tabla 13). 
Tabla 12. Clima. Matriz Factorial.

\begin{tabular}{|l|l|l|}
\hline & MATRIZ & COMUNALIDADES \\
\hline Cohesión & FACTORIAL & \\
Cooperación & 0,76 & 0,57 \\
Desarrollar personas y equipos & 0,75 & 0,56 \\
Autonomía & 0,68 & 0,47 \\
Relaciones sociales & 0,66 & 0,44 \\
Apoyo & 0,65 & 0,42 \\
Claridad & 0,65 & 0,42 \\
Grado de Estructura Impuesta sobre la posición & 0,64 & 0,64 \\
ocupada & & 0,40 \\
Implicación & 0.62 & \\
Flexibilidad/Innovación & 0,59 & 0,39 \\
Motivación & 0.53 & 0,35 \\
Confort & 0,52 & 0,28 \\
Centralización & 0,46 & 0,27 \\
Retribución & 0,43 & 0,21 \\
Estructura. & 0,41 & 0,19 \\
Orientación hacia la Recompensa & 0,38 & 0,16 \\
Presión & 0,33 & 0,15 \\
Control & $<0,3$ & 0,11 \\
Estatus & $<0,3$ & 0,07 \\
\hline Porcentaje de Varianza explicada & 34,2 & 0,02 \\
\hline
\end{tabular}

Tabla 13. Clima. Índice de Fiabilidad.

\begin{tabular}{|c|c|c|}
\hline INDICADOR & $\begin{array}{c}\text { CORRELACIÓN } \\
\text { INDICADOR TOTAL }\end{array}$ & $\begin{array}{c}\text { ALFA } \\
\text { PRESCINDIENDO } \\
\text { DEL INDICADOR }\end{array}$ \\
\hline Cohesión & 0,68 & 0,87 \\
\hline Cooperación & 0,70 & 0,87 \\
\hline Desarrollar personas y equipos & 0,62 & 0,87 \\
\hline Autonomía & 0,59 & 0,87 \\
\hline Relaciones sociales & 0,59 & 0,87 \\
\hline Apoyo & 0,60 & 0,87 \\
\hline Claridad & 0,59 & 0,87 \\
\hline $\begin{array}{l}\text { Grado de Estructura Impuesta sobre la posición } \\
\text { ocupada }\end{array}$ & 0,58 & 0,87 \\
\hline Implicación & 0,57 & 0,87 \\
\hline Flexibilidad/Innovación & 0,54 & 0,87 \\
\hline Motivación & 0,49 & 0,88 \\
\hline Confort & 0,47 & 0,88 \\
\hline Centralización & 0,44 & 0,88 \\
\hline Retribución & 0,42 & 0,88 \\
\hline Estructura. & 0,42 & 0,88 \\
\hline Orientación hacia la Recompensa & 0,39 & 0,88 \\
\hline Presión & 0,39 & 0,88 \\
\hline Control & 0,29 & 0,88 \\
\hline Estatus & 0,23 & 0,88 \\
\hline N de casos válidos: & & \\
\hline $\mathrm{N}$ de indicadores: & & \\
\hline Alfa & & \\
\hline
\end{tabular}

Dimensión 5. Cultura.

La media de las medias de los indicadores de esta dimensión arroja un valor de 7, 17. De los 11 indicadores que componen la misma, 9 cumplen con alguna de las condiciones de «pertenencia» y «atribución». La utilización del criterio de «explicación» (tabla 14) pone en evidencia que el indicador «Control» queda excluido del baremo de decisión establecido «0,30). 
Tabla 14. Cultura. Análisis Factorial

\begin{tabular}{|l|l|l|}
\hline & \multicolumn{1}{|c|}{$\begin{array}{c}\text { MATRIZ } \\
\text { FACTORIAL }\end{array}$} & COMUNALIDADES \\
\hline Dirección & 0.76 & 0,58 \\
Comunicación & 0,67 & 0,45 \\
Tolerancia al Conflicto & 0,63 & 0,40 \\
Autonomía & 0,63 & 0,39 \\
Apoyo & 0,62 & 0,38 \\
Coordinación & 0,61 & 0,38 \\
Tolerancia del Riesgo & 0,57 & 0,33 \\
Identidad & 0,53 & 0,28 \\
Sistema de Recompensa & 0,47 & 0,22 \\
Presunciones Básicas Subyacentes & 0,40 & 0,16 \\
Control & $<0,3$ & 0,06 \\
\hline Porcentaje de Varianza explicada & 38,6 & 33.0 \\
\hline
\end{tabular}

Por otro lado, el Índice de fiabilidad mejora si se suprimen los indicadores «Presunciones básicas subyacentes» $\mathrm{y}$ «Control» (tabla 15$)$.

Tabla 15. Cultura. Índice de Fiabilidad.

\begin{tabular}{|l|l|l|}
\hline \multicolumn{1}{|c|}{ INDICADOR } & $\begin{array}{c}\text { CORRELACIÓN } \\
\text { INDICADOR TOTAL }\end{array}$ & $\begin{array}{c}\text { ALFA PRESCINDIENDO } \\
\text { DEL INDICADOR }\end{array}$ \\
\hline Dirección & 0,70 & 0,80 \\
Comunicación & 0,60 & 0,81 \\
Tolerancia al Conflicto & 0,56 & 0,81 \\
Autonomía & 0,57 & 0,81 \\
Apoyo & 0.57 & 0,81 \\
Coordinación & 0.57 & 0,81 \\
Tolerancia del Riesgo & 0,52 & 0,81 \\
Identidad & 0,51 & 0,82 \\
Sistema de Recompensa & 0,42 & 0,82 \\
Presunciones Básicas Subyacentes & 0,37 & 0,84 \\
Control & 0,25 & \\
\hline N de casos válidos: 145 & \\
N de indicadores: $\quad 11$ & \\
Alfa & \multicolumn{2}{|l}{} \\
\hline
\end{tabular}

\section{Dimensión 6. Satisfacción.}

La media de las medias de los indicadores que componen esta dimensión es el más elevado: 7,62. De los 16 indicadores, hay 14 que cumplen alguno de los criterios de «atribución» o «pertenencia». La aplicación del criterio de «explicación» muestra que los indicadores «Especialización del trabajo» $\mathrm{y}$ «Estatus» quedan excluidos del mismo (tabla 16). Así mismo, el Índice de «fiabilidad» se incrementa si se eliminan estos dos mismos indicadores «Especialización en el trabajo» $\mathrm{y}$ «Estatus» (tabla 17). 
Tabla 16. Satisfacción. Análisis Factorial.

\begin{tabular}{|l|l|l|}
\hline & $\begin{array}{c}\text { MATRIZ } \\
\text { FACTORIAL }\end{array}$ & COMUNALIDADES \\
\hline Desarrollar Personas y Equipos & 0,74 & 0,55 \\
Dirección & 0,72 & 0,51 \\
Autonomía & 0,70 & 0,50 \\
Equidad & 0,69 & 0,47 \\
Apoyo & 0,68 & 0,46 \\
Iniciativa & 0,66 & 0,43 \\
Relaciones sociales & 0,65 & 0,43 \\
Comunicación & 0,62 & 0,38 \\
Evaluación del Rendimiento & 0,50 & 0,25 \\
Confort & 0,50 & 0,25 \\
Capacitación & 0,49 & 0,24 \\
Sistema de Recompensa & 0,47 & 0,22 \\
Retribución & 0,47 & 0,22 \\
Administración de Políticas & 0,47 & 0,22 \\
Especialización del Trabajo & $<0,3$ & 0,06 \\
Estatus & $<0,3$ & 0,01 \\
\hline Porcentaje de Varianza explicada & 36,2 & 32,5 \\
\hline
\end{tabular}

Tabla 17. Satisfacción. Índice de Fiabilidad.

\begin{tabular}{|c|c|c|}
\hline & $\begin{array}{c}\text { MATRIZ } \\
\text { FACTORIAL } \\
\end{array}$ & COMUNALIDADES \\
\hline Desarrollar Personas y Equipos & 0.67 & 0,85 \\
\hline Dirección & 0.67 & 0,85 \\
\hline Autonomía & 0.64 & 0,85 \\
\hline Equidad & 0.64 & 0,86 \\
\hline Apoyo & 0.62 & 0,86 \\
\hline Iniciativa & 0.60 & 0,86 \\
\hline Relaciones sociales & 0.62 & 0,86 \\
\hline Comunicación & 0.58 & 0,86 \\
\hline Evaluación del Rendimiento & 0.49 & 0,86 \\
\hline Confort & 0.47 & 0,86 \\
\hline Capacitación & 0.47 & 0,86 \\
\hline Sistema de Recompensa & 0.44 & 0,86 \\
\hline Retribución & 0.44 & 0,86 \\
\hline Administración de Políticas & 0.43 & 0.87 \\
\hline Especialización del Trabajo & 0.25 & 0,87 \\
\hline Estatus & 0.11 & 0,88 \\
\hline $\mathrm{N}$ de casos válidos: & & \\
\hline $\mathrm{N}$ de indicadores: & & \\
\hline Alfa & & \\
\hline
\end{tabular}

\section{Dimensión 7. Eficacia.}

La media de las medias de los indicadores integrados en esta dimensión es de 7, la. Así mismo, de los 29 indicadores que componen la misma, 22 cumplen alguno de los criterios de «pertenencia»y «atribución». La aplicación del criterio de «explicación»subraya que los indicadores «Control»y «Estabilidad» deben ser excluidos de esta dimensión (tabla 18). Por otro lado, también la eliminación de dichos indicadores (<<Control» y «Estabilidad») mejora el coeficiente alfa de «fiabilidad» (tabla 19). 
Tabla 18. Eficacia. Análisis Factorial.

\begin{tabular}{|c|c|c|}
\hline & $\begin{array}{c}\text { MATRIZ } \\
\text { FACTORIAL }\end{array}$ & COMUNALIDADES \\
\hline Cohesión & 0,74 & 0,55 \\
\hline Flexibilidad/Adaptación & 0,734 & 0,55 \\
\hline Internalización de Objetivos de la Organización & 0,70 & 0,49 \\
\hline Desarrollar Personas y Equipos & 0,68 & 0,47 \\
\hline Utilización del entorno & 0,66 & 0,43 \\
\hline Participación e Influencia Compartida & 0,65 & 0,42 \\
\hline Crecimiento & 0,65 & 0,42 \\
\hline Valor de los Recursos Humanos & 0,64 & 0,41 \\
\hline Calidad & 0,62 & 0,39 \\
\hline Habilidades de Trabajo de la Dirección & 0,61 & 0,37 \\
\hline Satisfacción en el Trabajo & 0,61 & 0,37 \\
\hline Énfasis en el Logro & 0,60 & 0,37 \\
\hline Consenso en los objetivos & 0,58 & 0,34 \\
\hline Eficiencia & 0,58 & 0,34 \\
\hline Comunicación & 0,58 & 0,33 \\
\hline Motivación & 0,57 & 0,33 \\
\hline Planificar & 0,57 & 0,32 \\
\hline Absentismo & 0,47 & 0,22 \\
\hline Conflicto & 0,47 & 0,22 \\
\hline Valoración por Entidades Externas & 0,46 & 0,21 \\
\hline Habilidades Interpersonales de la Dirección & 0,46 & 0,21 \\
\hline Rotación & 0,44 & 0,19 \\
\hline Estructura & 0,42 & 0,18 \\
\hline Beneficio & 0,42 & 0,17 \\
\hline Accidentabilidad & 0,40 & 0,16 \\
\hline Moral & 0,39 & 0,16 \\
\hline Productividad & 0,35 & 0,12 \\
\hline Control & $<0,3$ & 0,03 \\
\hline Estabilidad & $<0,3$ & 0,01 \\
\hline Porcentaje de Varianza explicada & 32,4 & 30,2 \\
\hline
\end{tabular}


Tabla 19. Eficacia. Índice de Fiabilidad.

\begin{tabular}{|c|c|c|}
\hline INDICADOR & $\begin{array}{c}\text { CORRELACIÓN } \\
\text { INDICADOR TOTAL }\end{array}$ & $\begin{array}{c}\text { ALFA PRESCINDIENDO DEL } \\
\text { INDICADOR }\end{array}$ \\
\hline \multirow{2}{*}{$\begin{array}{l}\text { Internalización de Objetivos de la Organización } \\
\text { Flexibilidad/Adaptación }\end{array}$} & 0.66 & 0,92 \\
\hline & 0.69 & 0,92 \\
\hline Utilización del entorno & 0.65 & 0,92 \\
\hline Crecimiento & 0.63 & 0,92 \\
\hline Desarrollar Personas y Equipos & 0.63 & 0,92 \\
\hline Calidad & 0.64 & 0,92 \\
\hline Comunicación & 0.59 & 0,92 \\
\hline Participación e Influencia Compartida & 0.60 & 0,92 \\
\hline Énfasis en el Logro & 0.58 & 0,92 \\
\hline Eficiencia & 0.57 & 0,92 \\
\hline Absentismo & 0.48 & 0,92 \\
\hline Habilidades Interpersonales de la Dirección & 0.45 & 0,92 \\
\hline Conflicto & 0.45 & 0,92 \\
\hline Valoración por Entidades Externas & 0.45 & 0,92 \\
\hline Estructura & 0.42 & 0,92 \\
\hline Rotación & 0.43 & 0,92 \\
\hline Beneficio & 0.41 & 0,92 \\
\hline Moral & 0.41 & 0,92 \\
\hline Accidentabilidad & 0.41 & 0,92 \\
\hline Productividad & 0.36 & 0,92 \\
\hline Control & 0.16 & 0,92 \\
\hline Estabilidad & 0.12 & 0.92 \\
\hline
\end{tabular}

\section{CONCLUSIONES}

Se han identificado y aislado 49 indicadores de la representación organizacional. Estos 49 indicadores se van a tratar como las variables independientes utilizadas para el estudio de la organización como construcción social. Su inminente despliegue en elementos (ítems) medibles mediante técnicas escalares constituyen la base informativa del cuestionario CREO@.

En la elección final de los indicadores se ha optado por una postura conservadora: se han seleccionado aquellos que satisfacen, al menos, alguno de los criterios de decisión empleados en la dimensión empírica (atribución, pertenencia). A tal respecto, debe recordarse que los indicadores han sido evaluados por los expertos en función de la importancia asignada a los mismos en la «Representación Organizacional». De ahi que se haya optado por no falsear la percepción de tales expertos, por lo que la dimensión empírica ha sido decisiva en la elección final de los indicadores ${ }^{6}$. De cualquier modo, de los 49 indicadores seleccionados: 41 cumplen los cuatro criterios de decisión empleados y 8 cumplen con algunos de los criterios de decisión. A continuación, se presentan en la tabla 20 el listado de los indicadores seleccionados ordenados de mayor a menor según las puntuaciones media.

El análisis específico de las siete dimensiones teóricas que componen el conjunto de indicadores potenciales utilizados (setenta y nueve), pone en evidencia que las dimensiones Contexto y Estructura son escasamente relevantes. Ambas dimensiones son evaluadas por los expertos como menos importantes, cara a la Representación Organizacional, que el resto de dimensiones. Así mismo, en cada una de estas dimensiones sólo un indicador de las mismas satisface alguno de los criterios de inclusión empleados («Tecnología» en la dimensión Contexto y «Profesionalización» en la dimensión Estructura). Por tal razón, los criterios finalmente seleccionados atienden a las siguientes dimensiones teóricas: Procesos organizacionales, Clima, Cultura, Satisfacción, Eficacia. Las dimensiones que se utilizan para diseñar la organización no tienen importancia en la imagen que tienen los trabajadores de su entidad.

Por último, se quiere comentar que se hipotetizan diferencias entre los grupos de expertos: esto dio lugar a un análisis para contrastar tal hipótesis. 
Tabla 20. Selección de indicadores de la representación organizacional.

Tabla 20. Selección de indicadores de la representación organizacional.

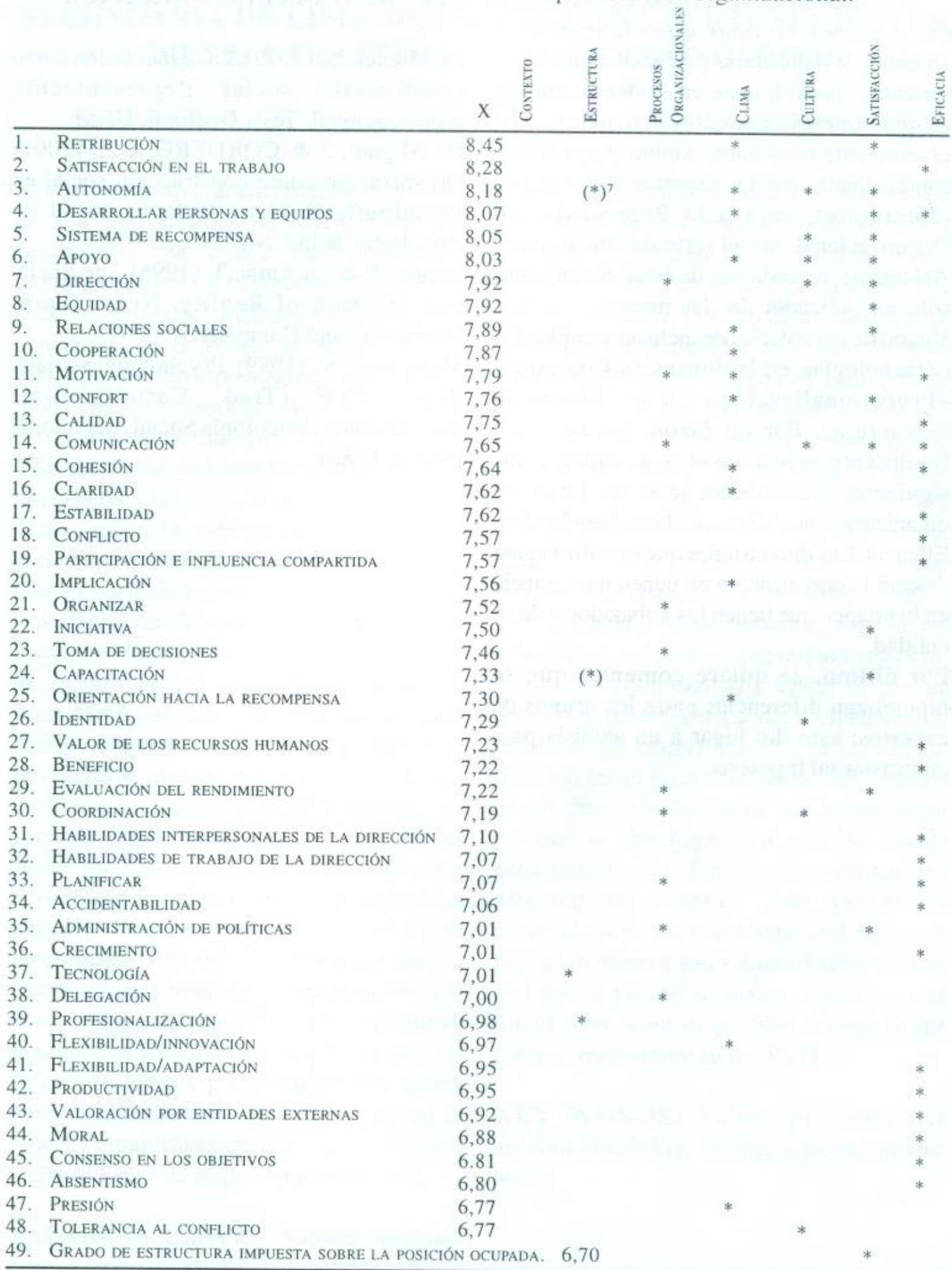

"Se ha optado por hacer una excepción a tal principio. El indicador «Presión» ha sido incluido en la lista de seleccionados a pesar de no satisfacer ninguno de los dos criterios de la dimensión empírica, aunque si cumple los dos criterios de explicación y fiabilidad. La decisión última ha estado motivada por el deseo del autor de incluir una variable que interesaba evaluar en una de las organizaciones donde se iba a realizar la aplicación del Cuestionario $\mathrm{CREO}^{\circ}$. La pertinencia de tal decisión ha sido después confirmada en un estudio posterior, siendo éste un indicador significativo dentro de las funciones discriminantes canónicas.

${ }^{2}$ Se han señalado como (*) aquellos indicadores que originalmente pertenecían a una dimensión y han sido excluidos de la misma por el análisis. Concretamente autonomía y capacitación se excluyen de estructura 


\section{REFERENCIAS BIBLIOGRAFICAS}

De Miguel, J.;. (1999). La organización como construcción social. Representación organizacional. Tesis Doctoral. UNM.

De Miguel, J \& CORDERO, c..;. (2001) Organización como construcción social en Psicología. Revista de la Facultad de Psicología de la UNMSM. p.55-58

Berger, P \& Luckman,T. (1996) The Social Construction of Reality. Nueva York. Doubleday and Company.

Moscovici, S. (1989) Psychology Sociale, Paris: PUF (Trad. Castellano de Rosembaunm: Psicología Social. Barcelona: Paidos, 1986. 